\title{
Flow Visualization of Sloshing in an Accelerated Two-Dimensional Rectangular Tank
}

\author{
Gurhan Sahin, Seyfettin Bayraktar* \\ Department of Naval Architecture \& Marine Engineering, Faculty of Naval Architecture \& Maritime, \\ Yildiz Technical University, 34349 Istanbul, Turkey \\ (sahin.gurhan@gmail.com, sbay@yildiz.edu.tr) \\ ॠCorresponding Author; Seyfettin Bayraktar, Yildiz Technical University, Department of Naval Architecture \& Marine \\ Engineering, Faculty of Naval Architecture \& Maritime, 34349 Istanbul, Turkey, Tel: +90 212383 7070, \\ sbay@yildiz.edu.tr
}

Received: 31.07.2015 Accepted:11.09.2015

\begin{abstract}
In the present paper, sloshing in a Two-Dimensional (2D) square liquid tank subjected to horizontal excitation is investigated by means of Computational Fluid Dynamics (CFD) technique. The tank with/out the vertical and horizontal baffles located at each side walls of the tank is moved on positive (+) x-axis for the excitation of $4.5 \mathrm{~m}^{2} / \mathrm{s}$ for each case study. After series of simulations results obtained for each tank configuration are compared and flow is visualized for the tanks that are $50 \%$ filled with the fresh water.
\end{abstract}

Keywords: Computational fluid dynamics (CFD), turbulence, sloshing, fluid, accelerated tank

\section{Introduction}

Sloshing is an important engineering problem. It may cause large deformations to wall and supporting structures in partially filled tanks. According to the classification societies`guidance sloshing may be defined as violent behavior of the liquid contents in tanks [1]. The phenomenon can be seen in many industries including automotive, aerospace, motorcycle, shipbuilding and maritime from the sloshing oscillations in aircrafts or space-crafts to storage tanks of ocean-going ships [2, 3].

Different wave conditions in partially filled tanks, uncontrolled loading/unloading processes, structural frequencies, shape and position of the tank, sources of the motions, filling levels inside the tanks, density of the fluid, etc. may cause sloshing. The tanks may be rectangular, prismatic, tapered, spherical and cylindrical. The carried liquids may be oil, liquefied gas, water, molasses and caustic soda [4]. All these above parameters show how the sloshing is complex and difficult to analyze. As stated by Rudman and Cleary [5] sloshing affects ship stability and therefore, great attention must be paid during not also loading and/or unloading period but also transportation. Due to demand of sloshing analysis for building large Liquefied Natural Gases (LNG) carriers and LNG platforms classification societies publish rules and guidance on this issue. For example, Bureau Veritas [1] and Det Norske Veritas [6] show the importance and specify the basic requirements for approval of LNG carriers and floating structures as well as provide necessary methodology to assess the sloshing loads and how to use these methodologies. One may ask why tanks are left partially-filled. The reason is that partial fillings in LNG carriers are a consequence of boil-off of gas during operations [4]. Up to now many experimental and numerical works have been performed. Krata [7] presented the results of an experimental and numerical works of a tank filled partially with the water. The pressure was measured while the tank was oscillated with the amplitude of $18^{\circ}, 30^{\circ}$, and $40^{\circ}$ which reflects the worst heavy sea conditions. Results showed that the measured pressure consists of non-impulsive dynamic pressure and impulsive (impact) pressure. The first one varies slowly due to the global movement of the liquid in the tank while the second lasts shortly and due to the hydraulic jump. An improved volume of fluid (VOF) model was developed by Wemmenhove et al. [8] and the numerical results were compared by a 1:10 model test. Although no any details on computational approach were given it was claimed that both numerical and experimental results were in a good agreement. Hou et al. [9] imposed external single and multiple excitations to the tank and analyzed the sloshing by CFD technique. It was revealed that the sloshing effects 
increased when the numbers of coupled excitations were added. Shoji and Munakata [10] tried to analyze the sloshing due to an earthquake by means of Fluid-Structure Interaction (FSI) and their results cleared that the current potential theories does not agree so much with the FSI analyses. Lots of methods have been employed to analyze the sloshing characteristics such as quasi-static method, hydrodynamics method, experimental method, equivalent mechanical method and computer simulation. Interested readers may find detailed knowledge in the work of Xue-Lian et al. [11]. Each technique has some advantages and disadvantages and among them experimental and computational techniques take more interests.

One of the fuel tank design objectives is to effectively reduce noise level caused by fluid motion inside the tank by designing baffles and separators to control the sloshing. In addition, alternate materials and manufacturing processes are evaluated for fuel tank design in order to reduce weight and cost and to provide structural integrity for higher structural performance. Sloshing in the tank may be controlled by incorporating baffles, and the effectiveness highly depends on the shape, the location, and the number of baffles inside a tank.

In the present paper, one of the test cases of Akyildiz and Celebi [12] and Javanshir et al. [13] is inspired where a rectangular tank was separated into mainly three regions by means of a vertical and two horizontal baffles. In addition to these types of configurations, a new one is introduced in the present paper and analyzed by means of CFD. The horizontal baffles that connected to the left and rights walls of the tanks are raised $15^{\circ}$ upwards.

\section{Figures and Tables}

It is obvious that sloshing occurs due to motion of fluid inside partially filled moving tank. As a passive control method baffles can be used to reduce the severe effects of sloshing. The following case studies simulate sloshing in a partially filled rigid tanks with and without the baffles and report the results for each configurations.

\subsection{Tank Configurations}

Rectangular tank with $250^{\mathrm{mm}} \times 250^{\mathrm{mm}}$ dimensions are considered to evaluate the performance of sloshing. The tanks with three baffle configurations are filled $50 \%$ with fresh water. The dimensions of the tank and baffles are showed in Fig.1. As shown in Fig.1a the first tank do not have any baffles while the second type tank has two horizontal baffles which are parallel to the bottom and a vertical baffle is in the middle of bottom (Fig.1b). Horizontal baffles of third type are angled $15^{\circ}$ upward while the vertical baffle is kept constant in the middle of the bottom (Fig.1c). All type of tanks are investigated according to below assumptions:

- Tanks are moving with $4.5 \mathrm{~m} / \mathrm{s}^{2}$ acceleration in $+\mathrm{x}$ direction (in 0-2 s)
- Tanks are moving with $4.5 \mathrm{~m} / \mathrm{s}^{2}$ acceleration in $-\mathrm{x}$ direction (in 2-4 s)

- Tanks are moving with $4.5 \mathrm{~m} / \mathrm{s}^{2}$ acceleration in $+x$ direction (in 4-6 s)

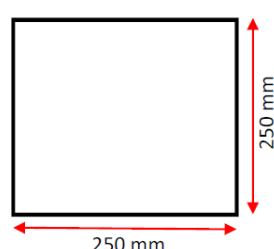

(a)

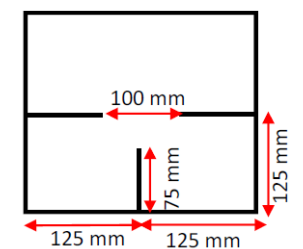

(b)

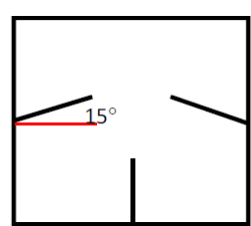

(c)
Fig. 1. Tank configurations, a) type-1, b) Type-2, c) Type-3

\subsection{Mesh Structure}

Number of mesh elements, aspect ratios and skewness that show quality of the mesh structure is summarized in Table.1.

Table 1. Mesh information of each configuration

\begin{tabular}{|c|c|c|c|}
\hline & $\begin{array}{c}\text { Number of } \\
\text { elements }\end{array}$ & $\begin{array}{c}\text { Maximum } \\
\text { aspect ratio }\end{array}$ & $\begin{array}{c}\text { Maximum } \\
\text { skewness }\end{array}$ \\
\hline Type-1 tank & 50690 & 1.2896 & 0.410 \\
\hline Type-2 tank & 65528 & 1.9586 & 0.573 \\
\hline Type-3 tank & 65302 & 1.8882 & 0.622 \\
\hline
\end{tabular}

As shown in Fig.2, different mesh elements are used for each tank configuration. Only quadrilateral elements are used for Type-1 tank while hybrid elements (quadrilateral and dominantly triangles) are preferred for Type- 2 and Type- 3 tanks.

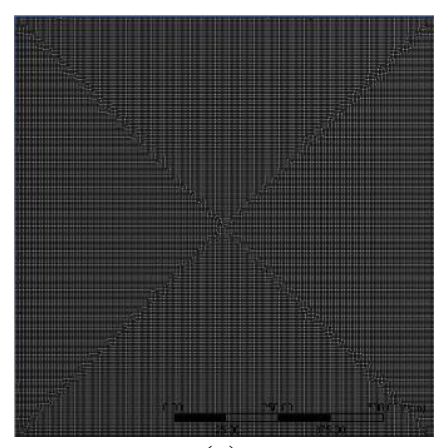

(a)

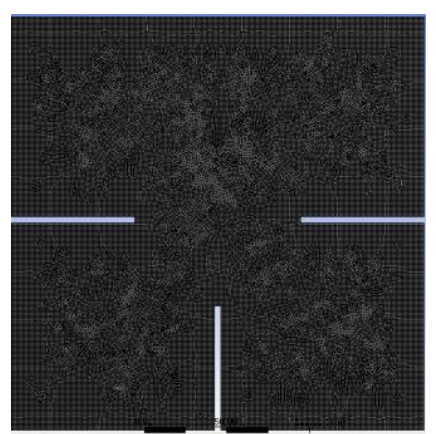

(b)

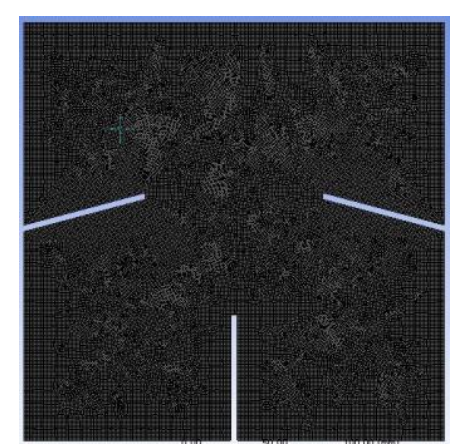

(c)

Fig. 2. Mesh structure of a) Type-1, b) Type-2, c) Type-3 


\section{Mathematical Model}

The equations used to simulate the sloshing are the continuity (Eq.1), Navier-Stokes (Eq.2) and VOF equations. These are given in general form as follows:

$\nabla \vec{V}=0$

$\frac{D \vec{V}}{D t}+-\frac{1}{\rho}[\nabla p-\mu(\vec{\nabla} \vec{\nabla}) \vec{V}]+\vec{F}_{B}$

The momentum equation is dependent on the volume fractions of all phases and given in Eq.3

$$
\frac{\partial u}{\partial t}+(\vec{V} \vec{\nabla}) \vec{V}=-\frac{1}{\rho}(\nabla p-\mu(\vec{\nabla} \vec{\nabla}) \vec{V})+F_{B}+F_{V}
$$

where $\vec{V}$ is the fluid velocity relative to tank, $\mathrm{p}$ is pressure; $\rho$ is fluid density and $\mu$ is viscosity. $F_{B}$ and $F_{v}$ state body force and virtual body force induced by the motion of tank.

As stated in detail by Nema [14] there are several techniques such as

- Moving grid or Lagrangian approach (capturing),

- Fixed grid or Eulerian approach (tracking),

- Combined method of Langrangian and Eulerian

for tracking immiscible interfaces. In the present study VOF, a part of Eulerian approach, has been used. The VOF model assumes that there is no any interaction between all the fluid phases (air and water here). For each control volume, the volume fraction of the phase in the cell will be introduced and the volume fraction of all the phases sums to unity. If $\alpha_{b}$ represent the both fluid's volume fraction in the cell, then the following three conditions are possible:

- $\alpha_{b}=0$ : Shows the cell is empty (no fluid of $b$ type is present)

- $\alpha_{b}=1$ : Shows the cell is full (only b type fluid is present)

- $0\left\langle\alpha_{b}\langle 1\right.$ : Shows the cell contain the interface between the both fluid and one or more other type of fluid.

Equation of volume fraction (VOF) for the both phase is given in Eq.4.

$\frac{1}{\rho}\left[\frac{\partial}{\partial t}\left(\alpha_{b} \rho_{b}\right)\right]+\vec{\nabla}\left(\alpha_{b} \rho_{b} \vec{V}_{b}\right)=s_{\alpha_{b}}+\sum\left(\dot{m}_{a b}-\dot{m}_{b a}\right)$

where $\dot{m}_{a b}, \dot{m}_{b a}$ and $s_{a b}$ is the mass transfer from a to b phase, mass transfer from $b$ to a phase and source term which permits the use of cavitation model. For $n$ phases:

$\sum_{b=1}^{n} \alpha_{b}=1$

Following equation is used to calculate physical parameters in the 2-phase flow for $\mathrm{a}$ and $\mathrm{b}$ :

$\rho=\alpha \rho_{b}+(1-\alpha) \rho_{a}$

$\mu=\alpha \mu_{b}+(1-\alpha) \mu_{a}$

Where $\alpha$ is defined as 1 for water and 0 for air.

As turbulence model, standard $\mathrm{k}-\varepsilon$ is selected that requires the solution of $\mathrm{k}$, turbulent kinetic energy (Eq.7) and $\varepsilon$, its dissipation rate (Eq.8).

$$
\begin{aligned}
& \frac{\partial k}{\partial t}+\frac{\left(k u_{j}\right)}{\partial x_{j}}=\frac{\partial}{\partial x_{j}}\left[\left(v+\frac{v_{t}}{\sigma_{k}}\right) \frac{\partial k}{\partial x_{j}}\right]+p_{k}-\varepsilon \\
& \frac{\partial \varepsilon}{\partial t}+\frac{\partial\left(\varepsilon u_{j}\right)}{\partial x_{j}}=\frac{\partial}{\partial x_{j}}\left[\left(v+\frac{v_{t}}{\sigma_{\varepsilon}}\right) \frac{\partial \varepsilon}{\partial x_{j}}\right]+C_{\varepsilon 1} P_{K} \frac{\varepsilon}{k}-C_{\varepsilon 2} \frac{\varepsilon^{2}}{k}
\end{aligned}
$$

where $\mathrm{P}_{\mathrm{K}}$ is the production of kinetic energy. Value of each coefficient seen in the equations are given in Table 2.

Table 2. Value of coefficients.

\begin{tabular}{|c|c|c|c|c|}
\hline$C_{\varepsilon 1}$ & $C_{\varepsilon 2}$ & $C_{\mu}$ & $\sigma_{k}$ & $\sigma_{\varepsilon}$ \\
\hline 1.44 & 1.92 & 0.09 & 1.0 & 1.3 \\
\hline
\end{tabular}

It is assumed that there is no slip on tank sides, bottom and top walls. The related equations are solved for time step of 0.01 second for all cases. Recommendations of Javanshir et al. [13] are followed initially. The model was validated by theory [15] and then baffles that are parallels and angled to the bottom of the thanks are used to reduce liquid sloshing.

\subsection{Linear Acceleration}

Initially, the tank is kept at rest and it is started to move along positive $(+) \mathrm{x}$-direction with constant acceleration of $4.5 \mathrm{~m} / \mathrm{s}^{2}$. The liquid is deviated from equilibrium state and then moved and clashed with the wall of the tank. After that water is in stable condition at a certain angle. Based on the theory, the free surface of liquid must be perpendicular to the pressure gradient and is thus tilted at a downward angle $(\theta)$ that is given in Eq.9 [15]:

$\theta=\operatorname{Arctan}\left(\frac{a_{x}}{a_{y}+\vec{g}}\right)$

where $\vec{g}, \mathrm{a}_{\mathrm{x}}$ and $\mathrm{a}_{\mathrm{y}}$ is the gravitational acceleration (in $\mathrm{y}$ direction), acceleration in $\mathrm{x}$-direction and finally acceleration in y-direction, respectively. Theoretically, $\theta$ is found as to be $24.64^{\circ}$. This value is validated by the present CFD study as shown in Fig.3.

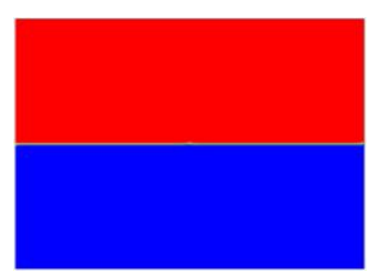

(a)

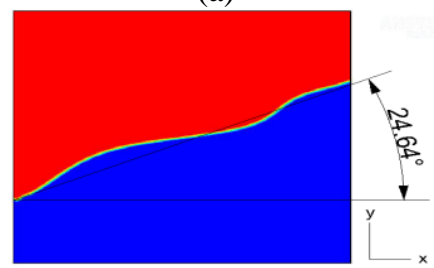

(c)

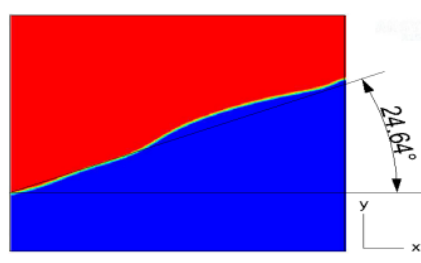

(b)

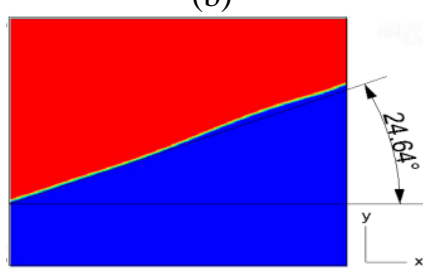

(d) 
Fig. 3. Sloshing in a rectangular tank without barrels under linear acceleration of $4.5 \mathrm{~m} / \mathrm{s}^{2}$ in the $(+) \mathrm{x}$-direction during a) $t=0, b) t=2$ seconds, c) $t=5.5$ seconds, $d$ ) $t=10$ seconds

\section{Results and Discussion}

Fig. 4 depicts the position of the free surface under the excitation for different configurations at various time from $\mathrm{T}=1$ second to $\mathrm{T}=6$ seconds. Since flow is excitated towards the $(+) \mathrm{x}$ direction at the beginning the water starts to move into that direction, impact on the right wall and then rises. With the gained energy it changes its direction and impacts on the left wall of the tank, then overturns and generates overturning waves (Fig.4a).

In the case of Type-2 tank the fluid (water) cannot rise so much. However, due to the presence of an obstructing object (horizontal baffle) it is disturbed and waves occured on top of the baffle. As time goes by some bubbles occured in the waves (Fig.4b) that play a role to damp the peak pressures of impact because the bubbles reduce the density of the fluid (water) and therefore, damps the pressure effects, [16]. In the present study, no any bubbles are seen around the corner of the tank ceiling but at the corner of the baffles.

It is not seen any wave impact on the side walls when the Type-3 tank is taken into account (Fig.4c). Fluid barely get out of the region created by raised horizontal baffles and could not reach to the side walls at the beginning. After a duration only small-sized waves occured on the top of the horizontal baffles. It can be concluded that the smaller tank size leads to the less impact of sloshing due to some reasons such as increased tank size that tends to increase the highest natural sloshing period and the internal structures dampen the fluid motions [4].

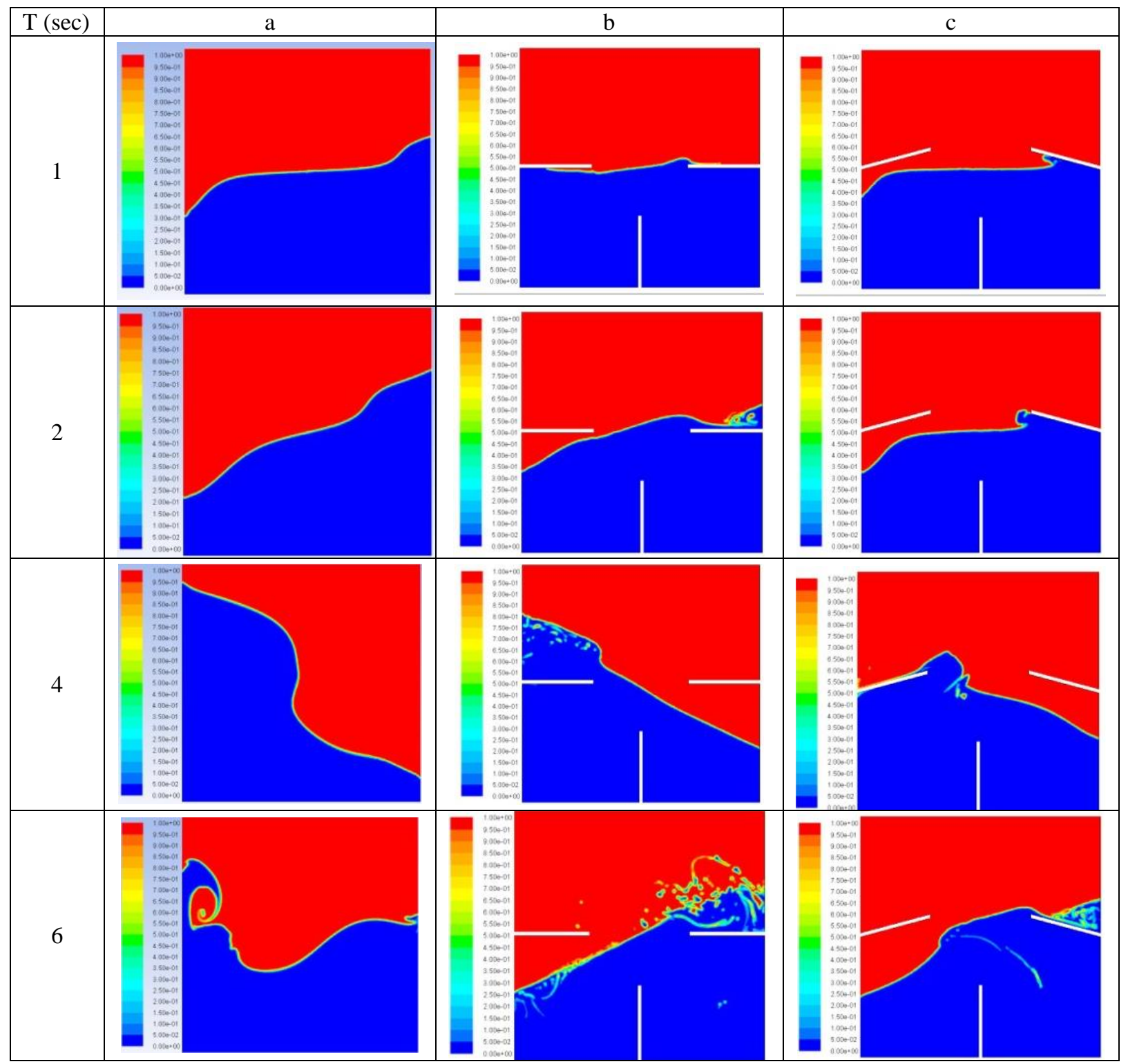

Fig. 4. Sloshing motion at different times for a) Type-1 tank, b) Type-2 tank and c) Type-3 type 
At the beginning, type-1 tank seems the worst while Type- 2 is the best because the movement of the water towards the left and right side walls can be prevented. Type-3 is better than Type-1, however, worse than Type-2. On the other hand, when time goes by Type- 3 tank prevents the sloshing and reduces its effects on side walls. In addition to this, Type- 2 prevents violent and overturning and breaking waves that reduce the effects of impact of water on the walls. Since the goal of the sloshing is to study the sloshing pattern and improve the tank design to reduce noise levels, stresses on the structure and optimize the baffle arrangements it can be concluded that Type- 3 is the most appropriate among others.

Fig.5 presents the turbulence kinetic energy (TKE) field. It is obvious that baffles distribute the TKE towards the center in a small region. The similar conclusion was drawn by Akyildiz and Celebi [12] and Celebi and Akyildiz [17] who investigated the effects of a vertical baffle in a rectangular tank. At the first seconds the TKE intensity is higher than the subsequent time and baffles concentrate the TKE towards the center of the tanks.

From the velocity vectors the direction of the movement of the fluid is clearly seen (Fig.6). During its excitation the water affects the air and enforces it to move in the direction of itself. At the beginning, a circulation region is generated and then it is distorted and its movement becomes irregular in Type-1 tank. A similar behavior of the fluid is seen in the other types of tanks but unlike the former one, four recirculation regions are formed below and over the horizontal baffles.

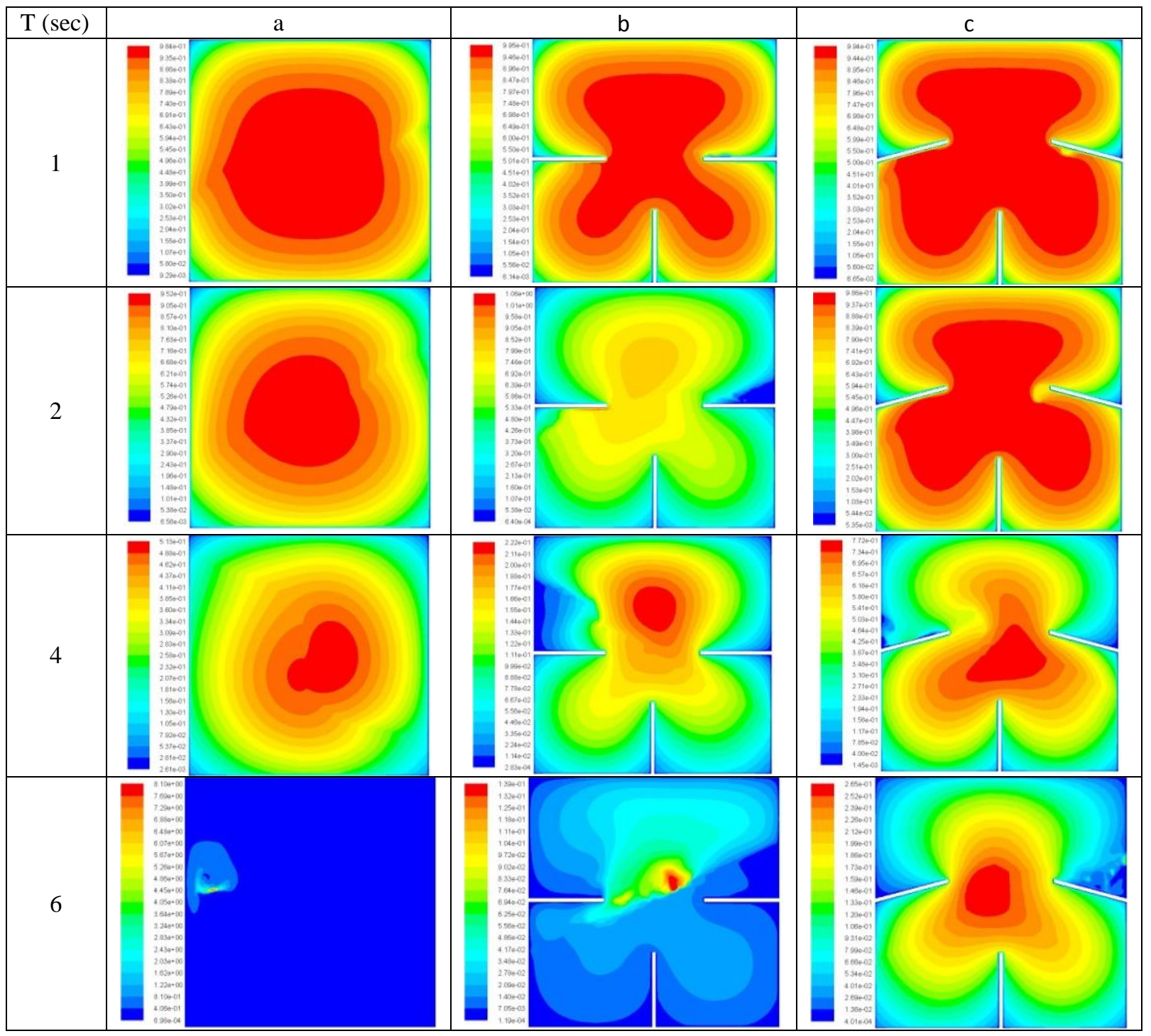

Fig. 5. Turbulence kinetic energy variations in a) Type-1 tank, b) Type-2 tank and c) Type-3 tank 


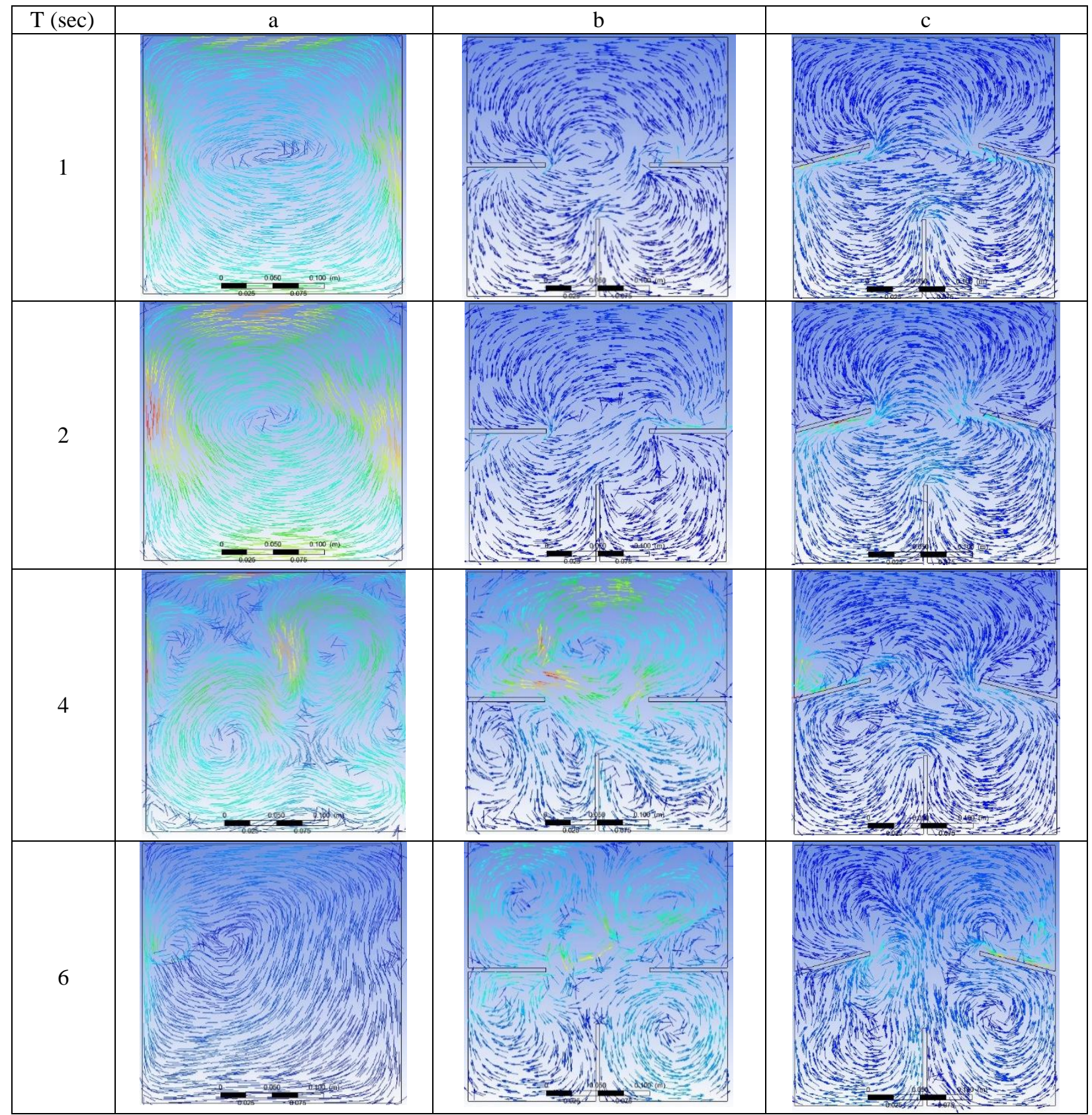

Fig. 6. Velocity vectors in a) Type-1, b) Type-2, c) Type-3

\section{Conclusion}

In the present CFD study, three types of tanks are analyzed under the same conditions. The computational results show that upward-angled baffles reduce the liquid sloshing amplitude than the other types. Type- 2 and Type- 3 is the configurations that the tanks is divided into mainly three regions; top, left and right regions. Although these type of configurations reduce the effects of sloshing it was concluded that most ship owners may not want to use such internal structures in cargo tanks because they may not enable the workers to easier cleaning. However, baffles prevents the rise of fluid towards the walls of the tank and therefore, reduces the sloshing effects that increases the life of the structural elements.

\section{References}

[1] Bureau Veritas, Guidance Note, Design Sloshing Loads for LNG Membrane Tanks, May 2011.

[2] Koli, G.C., Kulkarni, V.V., Simulation of Fluid Sloshing in a Tank, Proceeding of the World Congress on Engineering, WCE 2010, June 30-July 2, 2010.

[3] Wu, C., Chen, B., Transient Response of Sloshing Fluid in a Three Dimensional Tank, Journal of Marine Science and Technology, vol.20, no.1, pp.26-37.

[4] Faltinsen, O.M., Rognebakke, O.F., Effect of sloshing on ship motions, 16th International Workshop on Water Waves and Floating Bodies, Hiroshima, Japan, 2001.

[5] Rudman, M., Cleary, P.W., Modeling Sloshing in LNG Tanks, $7^{\text {th }}$ International Conference on CFD in the 
Minerals and Process Industries, CSIRO, Melbourne, Australia, 9-11 December 2009.

[6] Det Norske Veritas, Classification Notes on Sloshing Analysis if LNG Membrane Tanks, No.30.9, August 2014.Krata, P., Model of Interaction of Water and Tank`s Structure in Sloshing Phenomenon, International Journal of Marine Navigation and Safety of Sea Transportation, vol.2, no.4, December 2008.

[7] Krata, P, Model of Interaction of Water and Tank`s Structure in Sloshing Phenomenon, International Journal of Marine Navigation and Safety of Sea Transportation, Vol.2, No.4, 2008.

[8] Wemmenhove, R., Luppes, R., Veldman, A.E.P., Bunnik, T., Numerical Simulation and Model Experiments of Sloshing in LNG Tanks, International Conference on Computational Methods in Marine Engineering, MARINE 2007, Barcelona, Spain.

[9] Hou, L., Li, F., Wu, C., A Numerical Study of Liquid Sloshing in a Two-Dimensional Tank under External Excitations, J. Marine Sci. Appl., 11, 305, 310, 2012.

10] Shoji, Y., Munakata, H., Sloshing of Cylindrical Tank due to Seismic Acceleration, Abaqus Users Conference, Newport, Rhode Island, May 2008.

[11] Xue-Lian, Z., Xian-Sheng, L., Yuan-yuan, R., Equivalent Mechanical Model for Lateral Liquid
Sloshing in Partially Filled Tank Vehicles, Mathematical Problems in Engineering, vol.2012, article ID 162825, 2012.

[12] Akyildiz, H., Celebi, M.S., Numerical Computation of Hydrodynamic Loads on Walls of a Rigid Tank due to Large Amplitude Liquid Sloshing, Turkish J. of Eng. Sci., 26, 429-445, 2002a.

[13] Javanshir, A., Elahi, R., Passandideh-Fard, M., Numerical Simulation of Liquid Sloshing with Baffles in the Fuel Container, the $12^{\text {th }}$ Iranian Aerospace Society Conference, Amir Kabir University of Technology, 2013.

[14] Nema, P.K., Computational Study of Sloshing Behavior in 3-D Rectangular Tank with and without Baffle under Seismic Excitation, MSc Thesis, Department of Mechanical Engineering, National Institute of Technology, Rourkela, India, 2014.

[15] White, F.M., Fluid Mechanics, $5^{\text {th }}$ Edition, McGrawHill.

[16] Kim, Y., Experimental and Numerical Analyses of Sloshing Flows, Journal of Engineering Mathematics, vol.58, issue 1-4, pp.191-210, 2007.

[17] Celebi, M.S., Akyildiz, H., Nonlinear Modelling of Liquid Sloshing in a Moving Rectangular Tank, Ocean Engineering, vol.29, issue 12, pp.1527-1553, 2002b. 